\title{
Spectral theory of corrugated surfaces
}

\author{
Vojkan Jakšić
}

\begin{abstract}
We discuss spectral and scattering theory of the discrete Laplacian limited to a half-space. The interesting properties of such operators stem from the imposed boundary condition and are related to certain phenomena in surface physics.
\end{abstract}

\section{Introduction}

This paper is a brief review of the program initiated some years ago in [JMP]. The main goal of this program is to understand the formation and the propagation properties of surface states in regions with corrugated boundaries. The history of this problem and its physical aspects are discussed in [JMP, KP].

The results described in this review are obtained in collaborations with Yoram Last and Stanislav Molchanov. I am grateful to Leonid Pastur and Barry Simon for many useful discussions on the subject of this review.

\section{The model}

Let $d \geq 1$ be given and let $\mathbb{Z}_{+}^{d+1}:=\mathbb{Z}^{d} \times \mathbb{Z}_{+}$where $\mathbb{Z}_{+}=\{0,1, \ldots\}$. We denote the points in $\mathbb{Z}_{+}^{d+1}$ by $(n, x)$, for $n \in \mathbb{Z}^{d}$ and $x \in \mathbb{Z}_{+}$. Let $V: \mathbb{Z}^{d} \mapsto \mathbb{R}$ be a given function and let $H$ be the discrete Laplacian on $\mathcal{H}:=l^{2}\left(\mathbb{Z}_{+}^{d+1}\right)$ with boundary condition $\psi(n,-1)=V(n) \psi(n, 0)$. When $V=0$ the operator $H$ reduces to Dirichlet Laplacian which we denote by $H_{0}$. The operator $H$ acts as

$$
(H \psi)(n, x)= \begin{cases}\sum_{\left|n-n^{\prime}\right|_{+}+\left|x-x^{\prime}\right|=1} \psi\left(n^{\prime}, x^{\prime}\right) & \text { if } x>0 \\ \psi(n, 1)+\sum_{\left|n-n^{\prime}\right|_{+}=1} \psi\left(n^{\prime}, 0\right)+V(n) \psi(n, 0) & \text { if } x=0\end{cases}
$$

where $|n|_{+}=\sum_{j=1}^{d}\left|n_{j}\right|$. Physically, the boundary condition $V$ models the corrugated surface of the medium and one is interested in its effects on propagation properties of wave packets.

MSC 2000 : primary: 47N20, secondary: 35P25, 35R10, 39A70.

Keywords : surface states and waves, random potentials, spectral and scattering theory. 
Note that the operator $H$ can be also viewed as the Schrödinger operator

$$
H=H_{0}+V
$$

where the potential $V$ acts only along the boundary $\partial \mathbb{Z}_{+}^{d+1}=\left\{(n, 0): n \in \mathbb{Z}^{d}\right\}$. This point of view is notationaly convenient and we will adopt it in the sequel. We recall that the spectrum of $H_{0}$ is purely absolutely continuous and that

$$
\sigma\left(H_{0}\right)=[-2(d+1), 2(d+1)]
$$

The results we wish to describe fall into four categories.

1. Structural results. These results follow from the geometry of the model and hold for an arbitrary surface potential.

2. Random surface potentials. The surface potential is a random process on $\mathbb{Z}^{d}$. Such potentials describe effects of impurities distributed randomly along the surface of the medium.

3. Surface Maryland model. The surface potential is the Maryland potential $V(n)=\lambda \tan (\pi \alpha \cdot n+\theta)$. Such quasiperiodic potential may be used to describe strongly corrugated surfaces.

4. Surface states and spectra. These results deal with certain dynamical aspects of the model and are motivated by surface physics.

\section{Structural results}

Unless otherwise stated, all the results of this section hold for an arbitrary surface potential $V$.

Theorem 3.1 [JL1, CS] The wave operators

$$
\Omega^{ \pm}=\mathrm{s}-\lim _{t \rightarrow \pm \infty} \mathrm{e}^{\mathrm{i} t H} \mathrm{e}^{-\mathrm{i} t H_{0}}
$$

exist. In particular, $\sigma\left(H_{0}\right) \subset \sigma_{\mathrm{ac}}(H)$.

One can also compute the ranges of wave operators. For any $R \geq 0$ let $\Gamma_{R}:=$ $\left\{(n, x): n \in \mathbb{Z}^{d}, 0 \leq x \leq R\right\}, \Gamma_{\bar{R}}=\mathbb{Z}_{+}^{d+1} \backslash \Gamma_{R}$. We denote by $1_{R}, 1_{\bar{R}}$, the characteristic functions of the sets $\Gamma_{R}, \Gamma_{\bar{R}}$, and use the same symbol for the corresponding multiplication operators. Obviously, $1=1_{R}+1_{\bar{R}}$.

Theorem 3.2 [JL3]

$$
\begin{aligned}
\operatorname{Ran} \Omega^{ \pm} & =\left\{\psi: \forall R \geq 0, \int_{\mathbb{R}}\left\|1_{R} \mathrm{e}^{-\mathrm{i} t H} \psi\right\|^{2} \mathrm{~d} t<\infty\right\}^{\mathrm{cl}} \\
& =\left\{\psi: \forall R, k \geq 0,\left\|1_{R} \mathrm{e}^{-\mathrm{i} t H} \psi\right\|=O\left(\langle t\rangle^{-k}\right)\right\}^{\mathrm{cl}},
\end{aligned}
$$

where cl stands for the closure. 
Due to the free propagation along the $x$-axis, the above theorems are certainly expected. They are also relatively simply to prove. Subtler results are related to the question when and where the wave operators are complete and the spectrum of $H$ is purely absolutely continuous. To describe these results we need some additional notions.

Let

$$
\mathcal{H}^{\mathrm{s}}:=l^{2}\left(\Gamma_{0}\right) \equiv l^{2}\left(\mathbb{Z}^{d}\right), \quad \mathcal{H}^{\mathrm{b}}:=l^{2}\left(\Gamma_{\overline{0}}\right) .
$$

We will refer to these Hilbert spaces as the surface and the bulk. Clearly,

$$
\mathcal{H}=\mathcal{H}^{\mathrm{s}} \oplus \mathcal{H}^{\mathrm{b}}
$$

With respect to this decomposition the operator $H$ can be written as a $2 \times 2$ matrix

$$
H=\left[\begin{array}{ll}
H^{\mathrm{ss}} & H^{\mathrm{sb}} \\
H^{\mathrm{bs}} & H^{\mathrm{bb}}
\end{array}\right]
$$

Here, $H^{\mathrm{ss}}=h_{0}+V$, where $h_{0}$ is the usual discrete Laplacian on $l^{2}\left(\mathbb{Z}^{d}\right)$, and $H^{\mathrm{bb}}$ is the Dirichlet Laplacian on $l^{2}\left(\Gamma_{\overline{0}}\right)$. Note that $H^{\mathrm{bb}}$ is unitarily equivalent to $H_{0}$. The operators $H^{\mathrm{sb}}$ and $H^{\mathrm{bs}}$ couple the surface and the bulk, $H^{\mathrm{sb}}=\left(H^{\mathrm{bs}}\right)^{*}, H^{\mathrm{bs}} \delta_{(n, x)}=0$ if $x>0$ and $\left.H^{\text {bs }} \delta_{(n, 0)}\right)=\delta_{(n, 1)}$.

We will use notation similar to (3.2) for other operators on $\mathcal{H}$. For example, $1^{\text {ss }}$ is the projection onto the surface and $1^{\mathrm{bb}}$ is the projection onto the bulk.

For any $z \notin \sigma\left(H^{\mathrm{bb}}\right)$, we define

$$
\begin{aligned}
W_{\mathrm{s}}(z) & :=H^{\mathrm{sb}}\left(H^{\mathrm{bb}}-z 1^{\mathrm{bb}}\right)^{-1} H^{\mathrm{bs}}, \\
G_{\mathrm{s}}(z) & :=H^{\mathrm{ss}}-W_{\mathrm{s}}(z)-z 1^{\mathrm{ss}} .
\end{aligned}
$$

In physics literature, the operator $W_{\mathrm{s}}(z)$ is sometimes called the self-energy. Following [DJ], we will call $G_{\mathrm{s}}(z)$ the resonance function. Its importance stems from the identity

$$
1^{\mathrm{ss}}(H-z)^{-1} 1^{\mathrm{ss}}=G_{\mathrm{s}}^{-1}(z),
$$

which is a consequence of the Feshbach formula for $(H-z)^{-1}$. One can also show that the Hilbert space $\mathcal{H}^{\mathrm{s}}$ is a cyclic set for $H$. It then follows from (3.4) that the spectral properties of $H$ are encoded by the resonance function $G_{\mathrm{s}}(z)$.

In the sequel, we will use the short-hand $\mathbb{C}_{ \pm}:=\{z: \pm \operatorname{Im} z>0\}$. One can show (see [JL1]) that the operator-valued function $\mathbb{C}_{+} \ni z \mapsto W_{\mathrm{s}}(z)$ extends by continuity to a norm-continuous function on $\overline{\mathbb{C}}_{+}$. Thus, for $e \in \mathbb{R}$, we can define $G_{\mathrm{s}}(e)$ by (3.3). Clearly, $G_{\mathrm{s}}(e)$ is a closed operator with domain $\mathcal{D}(V)$. We define the resonant spectrum of the operator $H$ by

$$
\mathfrak{R}(H):=\left\{e \in \mathbb{R}: 0 \in \sigma\left(G_{\mathrm{s}}(e)\right)\right\} .
$$

If $V$ is a constant surface potential, $V=a$, then $\mathfrak{R}(H)=\emptyset$ if $|a|<1$ and

$$
\mathfrak{R}(H)=[-2 d, 2 d]+a+a^{-1}
$$

if $|a| \geq 1$. Motivated by this observation, we set

$$
\mathcal{S}(V):=\left\{[-2 d, 2 d]+a+a^{-1}: a \in \sigma(V),|a| \geq 1\right\} .
$$


One can show (see [JL1]) that

$$
\sigma(H) \subset \sigma\left(H_{0}\right) \cup \mathcal{S}(V)
$$

We also define

$$
\mathcal{S}_{\text {ext }}(V):=\mathcal{S}(V) \cup([-2 d, 2 d]+\{2 a: a \in \sigma(V),|a|<1\}) .
$$

The basic properties of the resonant spectrum are summarized in:

Theorem 3.3 [JL1]

(i) $\mathfrak{R}(H)$ is a closed set.

(ii) $\mathfrak{R}(H) \subset \sigma(H)$ and $\sigma(H) \backslash \sigma\left(H_{0}\right) \subset \mathfrak{R}(H)$.

(iii) $\mathfrak{R}(H) \subset \mathcal{S}_{\text {ext }}(V)$.

(iv) If $\|V\|<1$ then $\mathfrak{R}(H)=\emptyset$.

(v) If $\lim \sup |V(n)|<1$ or $\lim |V(n)|=\infty$ then $\mathfrak{R}(H)$ has Lebesgue measure 0 .

Theorem 3.4 [JL1] The wave operators $\Omega^{ \pm}$are complete on $\sigma(H) \backslash \mathfrak{R}(H)$. In particular, the spectrum of $H$ is purely absolutely continuous on this set.

The existing counterexamples [MV1, MV2] suggest that the last theorem is an optimal result in the sense that it holds for an arbitrary surface potential $V$.

\section{Random surface potentials}

Let

$$
\Omega=\mathbb{R}^{\mathbb{Z}^{d}}=\prod_{n \in \mathbb{Z}^{d}} \mathbb{R}
$$

be the set of all surface potentials, that is, the functions $V: \mathbb{Z}^{d} \mapsto \mathbb{R}$. Let $\mathcal{F}$ be the $\sigma$-algebra generated by the cylinder sets

$$
\left\{V: V\left(n_{1}\right) \in B_{1}, \ldots, V\left(n_{k}\right) \in B_{k}\right\},
$$

where $B_{1}, B_{2}, \ldots, B_{k}$ are Borel subsets of $\mathbb{R}$. The random surface model is specified by the choice of a probability measure $P$ on $(\Omega, \mathcal{F})$. Given $P$, one is interested in spectral and scattering properties of $H=H_{0}+V$ which hold $P$-a.s., that is, for a set of $V$ 's of $P$ measure 1.

We will consider measures $P$ of the form

$$
P=\prod_{n \in \mathbb{Z}^{d}} \mu_{n}
$$

where each $\mu_{n}$ is a probability measure on $\mathbb{R}$. Thus, $\mu_{n}$ is a probability distribution of the random variable $\Omega \ni V \mapsto V(n)$. The random variable $V(n)$ has density if the measure $\mu_{n}$ is absolutely continuous w.r.t. Lebesgue measure. It follows from the definition of $P$ that the random variables $\{V(n)\}$ are independent, and we say that they are i.i.d. if all the measures $\mu_{n}$ are equal to $\mu$. We recall that the topological 
support of $\mu, \operatorname{supp} \mu$, is the complement of the largest open set $B$ such that $\mu(B)=0$. Set

$$
\mathcal{S}:=\left\{[-2 d, 2 d]+a+a^{-1}: a \in \operatorname{supp} \mu,|a| \geq 1\right\} .
$$

Consider first the i.i.d. case. Then $H=H_{0}+V$ is an ergodic family of random operators and it follows from the well-known argument (see [CFKS]) that there exist closed sets $\Sigma, \Sigma_{\mathrm{ac}}, \Sigma_{\mathrm{pp}}, \Sigma_{\mathrm{sc}}$, such that $P$-a.s., $\sigma(H)=\Sigma, \sigma_{\mathrm{ac}}(H)=\Sigma_{\mathrm{ac}}$, $\sigma_{\mathrm{sc}}(H)=\Sigma_{\mathrm{sc}}$. Note that $P$-a.s. $\sigma(V)=\operatorname{supp} \mu$ and so

$$
\mathcal{S}(V)=\mathcal{S} \quad P-a . s .
$$

One can show (see [JL1] and compare with (3.5)) that

$$
\Sigma=\sigma\left(H_{0}\right) \cup \mathcal{S}
$$

Theorem 3.3 is complemented with the following result.

Theorem 4.1 Assume that the random variables $\{V(n)\}_{n \in \mathbb{Z}^{d}}$ are i.i.d. Then:

(i) There is a set $\mathfrak{R}$ such that $\mathfrak{R}(H)=\mathfrak{R} P$-a.s.

(ii) $\mathcal{S} \subset \mathfrak{R}$.

(iii) If $\operatorname{supp} \mu \cap(-1,1)=\emptyset$, then $\mathcal{S}=\mathfrak{R}$.

The above result yields that in many situations there is a rich resonant spectrum on $\sigma\left(H_{0}\right)$. The deterministic Theorem 3.4 gives no information about the structure of the spectrum on the set $\mathfrak{R} \cap \sigma\left(H_{0}\right)$ (by Theorem 3.1, though, we know that there is a component of a.c. spectrum on this set). The next result gives complete characterization of the spectrum of $H$ in $\sigma\left(H_{0}\right)$ if the random variables $V(n)$ have densities.

Theorem 4.2 [JL1, JL2] Assume that the random variables $\{V(n)\}_{n \in \mathbb{Z}^{d}}$ have densities. Then the spectrum of $H$ in $\sigma\left(H_{0}\right)$ is $P$-a.s. purely absolutely continuous.

Combining Theorems 3.3 and 4.2 one derives:

Corollary 4.3 [JL1] Assume that the assumption of Theorem 4.2 holds and that either

$$
\limsup _{|n| \rightarrow \infty}|V(n)|<1 P-\text { a.s. } \quad \text { or } \quad \lim _{|n| \rightarrow \infty}|V(n)|=\infty P-\text { a.s. }
$$

Then the wave operators $\Omega^{ \pm}$are complete on $\sigma\left(H_{0}\right)$.

Under the assumptions of the previous corollary, the spectrum of $H$ outside $\sigma\left(H_{0}\right)$ is $P$-a.s. discrete.

We proceed to describe the structure of the spectrum outside $\sigma\left(H_{0}\right)$. The first results in this direction were obtained in [AM, G]. Note that by the formula (4.1), in the i.i.d. case the operator $H$ will have $P$-a.s. some essential spectrum outside $\sigma\left(H_{0}\right)$ whenever $\operatorname{supp} \mu \backslash[-1,1] \neq \emptyset$.

For simplicity, until the end of this section we will assume that the random variables $\{V(n)\}$ are i.i.d. with distribution $\mathrm{d} \mu=p(x) \mathrm{d} x$. 
Theorem 4.4 [JM2] Assume that $d+1=2, p \in L^{\infty}(\mathbb{R})$, and that the topological boundary of $\operatorname{supp} \mu$ is a discrete set. Then the operator $H$ has P-a.s. only pure point spectrum outside $\sigma\left(H_{0}\right)$.

In [JM2] it is also shown that the eigenfunctions of $H$ decay exponentially in the $x$-variable and faster then any polynomial in the $n$-variable. We do not believe that this result is optimal (see Theorem 4.5 below).

Theorem 4.4 is a non-perturbative result and its proof is technically demanding. We outline the strategy of the proof. The operators $G_{\mathrm{s}}(e)$ can be written as

$$
G_{\mathrm{s}}(e)=h_{0}(e)+V-e,
$$

where $h_{0}(e)=h_{0}-W_{\mathrm{s}}(e)$, and $h_{0}$ is the usual discrete Laplacian on $l^{2}\left(\mathbb{Z}^{d}\right)$. The operator $h_{0}(e)$ acts as convolution by a certain function $j(n, e)$, that is,

$$
\left(h_{0}(e) \psi\right)(k)=\sum_{k} j(n-k, e) \psi(k) .
$$

We adopt the short-hand $h(e)=h_{0}(e)+V$. It is not difficult to show that $j(n, e)$ decays exponentially in the $n$-variable for $e \notin \sigma\left(H_{0}\right)$,

$$
|j(n, e)| \leq C \mathrm{e}^{-\gamma(e)|n|}
$$

The exponential decay is not uniform and $\gamma(e) \rightarrow 0$ as $|e| \downarrow 2(d+1)$.

Let $(a, b)$ be an interval outside $\sigma\left(H_{0}\right)$. The Simon-Wolff theorem [SW] can be adapted to the half-space problems and it yields that if for $\mathrm{d} e \otimes \mathrm{d} P$ a.e. pairs $(e, V) \in(a, b) \times \Omega$,

$$
\lim _{\epsilon \downarrow 0} \sum_{n}\left|\left(\delta_{0} \mid(h(e)-e-\mathrm{i} \epsilon)^{-1} \delta_{n}\right)\right|^{2}<\infty
$$

then the spectrum of $H$ is $P$-a.s. pure point on $(a, b)$. Therefore, one needs a suitable estimate on

$$
\left|\left(\delta_{0} \mid(h(e)-e-\mathrm{i} \epsilon)^{-1} \delta_{n}\right)\right|
$$

for a.e. $(e, V)$. In comparison with the usual theory of random Schrödinger operators, the difficulties in estimating (4.3) stem from the fact that $h_{0}(e)$ is long-range energy-dependent Laplacian.

When $d=1$, we have estimated (4.3) in [JM1, JM2] following geometric KAM approach to one-dimensional localization which goes back to [KMP, M1, M2, GJMS]. The heuristic ideas behind this estimation are explained in detail in the introductions of [JM1, JM2].

The method of [JM2] works only for $d=1$, and a new approach is needed in higher dimensions. This problem was studied in [JM3] using the AizenmanMolchanov technique. The basic observation is that although there is no uniform exponential bound on the sequence $j(n, e)$, the following bound holds:

$$
\sup _{e \notin \sigma\left(H_{0}\right)}|j(n, e)| \leq C \prod\left\langle n_{i}\right\rangle^{-(d+1) / d} .
$$


The estimates (4.2), (4.4) and Aizenman-Molchanov theory yield that the sequence (4.3) decays exponentially in $n$ for all $e \notin \sigma\left(H_{0}\right)$. We state the result of [JM3] under a simple assumption on the density $p$. The interested reader may consult the original paper for various generalizations.

Theorem 4.5 [JM3] Let $d \geq 1$ and $H=H_{0}+\lambda V, V \in \Omega$, where $\lambda$ is a real constant. Assume that $p(x)=O\left(\langle x\rangle^{-1-\alpha}\right)$ for some $\alpha>2 d /(d+1)$. Then there are constants $0<a_{d}<b_{d}$ such that for $|\lambda| \notin\left[a_{d}, b_{d}\right]$ the operator $H$ has $P$-a.s. only pure point spectrum outside $\sigma\left(H_{0}\right)$ with exponentially decaying eigenfunctions.

The weak coupling $\left(|\lambda|<a_{d}\right)$ and the large coupling $\left(|\lambda|>b_{d}\right)$ regime of Theorem 4.5 are mathematically and physically very different. A perhaps most important difference is that the large coupling regime allows for a background surface potential $U_{0}$, while the weak coupling regime does not. For a discussion of mathematical and physical reasons behaind this difference we refer the reader to [JM3].

\section{Surface Maryland model}

Let $\alpha=\left(\alpha_{1}, \cdots, \alpha_{d}\right) \in[0,1]^{d}$ and $\theta \in[0, \pi]$ be given. The Maryland potential on $\mathbb{Z}^{d}$ is the function

$$
V_{\alpha, \theta}(n)=\tan (\pi \alpha \cdot n+\theta) .
$$

To avoid singular cases, one assumes that for a given $\alpha, \theta$ is chosen so that for all $n$,

$$
\pi \alpha \cdot n+\theta \not \equiv 0 \bmod \pi / 2 .
$$

The results described in this section hold for all $\theta$ which satisfy this relation.

The usual Maryland model is a family of operators on $l^{2}\left(\mathbb{Z}^{d}\right)$ of the form $h_{\lambda, \alpha, \theta}=$ $h_{0}+\lambda V_{\alpha, \theta}$, where $\lambda$ is a real parameter and $h_{0}$ the discrete Laplacian on $l^{2}\left(\mathbb{Z}^{d}\right)$. This model has been introduced in physics literature by Fishman, Grempel and Prange in early 1980's, and has been extensively studied (see [CFKS] for references and additional information). We say that $\alpha=\left(\alpha_{1}, \cdots, \alpha_{d}\right)$ is independent over rationals if for any choice of rational numbers $r_{1}, \cdots, r_{d} \in \mathbb{Q}, \sum r_{k} \alpha_{k} \notin \mathbb{Q}$. We say that $\alpha$ has typical Diophantine property if there exist constants $C, k>0$ such that $|n \cdot \alpha-m|>C|n|^{-k}$ for all $n \in \mathbb{Z}^{d}, m \in \mathbb{Z}$. The set of such $\alpha$ 's in $[0,1]^{d}$ has Lebesgue measure 1. If $\alpha$ has typical Diophantine properties then for all $\lambda \neq 0$, $\sigma\left(h_{\lambda, \alpha, \theta}\right)=\mathbb{R}$, the spectrum is pure point, the eigenvalues of $h_{\lambda, \alpha, \theta}$ are simple and the corresponding eigenfunctions decay exponentially (see [CFKS, FP]).

The surface Maryland model is the family of operators on $l^{2}\left(\mathbf{Z}_{+}^{d+1}\right)$ defined by

$$
H_{\lambda, \alpha, \theta}=H_{0}+\lambda V_{\alpha, \theta},
$$

where $V_{\alpha, \theta}$ acts only along the boundary $\partial \mathbb{Z}_{+}^{d+1}=\mathbb{Z}^{d}$.

The surface Maryland model was first studied by Khoruzenko and Pastur. They have proven the following the result.

Theorem $5.1[\mathrm{KP}]$ Assume that $\alpha$ has typical Diophantine properties. Then, for all $\lambda \neq 0, \sigma\left(H_{\lambda, \alpha, \theta}\right)=\mathbb{R}$ and the spectrum of $H_{\lambda, \alpha, \theta}$ on the set $\mathbb{R} \backslash \sigma\left(H_{0}\right)$ is pure point. On this set the eigenvalues are simple and the corresponding eigenfunctions decay exponentially. 
It follows from Theorem 3.1 that $\sigma\left(H_{0}\right) \subset \sigma_{\mathrm{ac}}\left(H_{\lambda, \alpha, \theta}\right)$. The structure of the spectrum of $H_{\lambda, \alpha, \theta}$ in $\sigma\left(H_{0}\right)$ was studied in [JM1, JM4].

Theorem 5.2 [JM1, JM4] Assume that $\alpha$ is independent over rationals. Then, for all $\lambda$, the wave operators (3.1) are complete on $\sigma\left(H_{0}\right)$. In particular, the spectrum of $H$ in $\sigma\left(H_{0}\right)$ is purely absolutely continuous.

A perhaps surprising aspect of Theorem 5.2 is that the scattering is complete without any Diophantine restrictions on $\alpha$. If $\alpha$ is dependent over rationals, the potential $V$ is periodic and the wave operators might not be complete.

For a discussion of the surface Maryland model in the context of surface physics we refer the reader to [KP, JM4].

In a recent work, Bentosela, Briet and Pastur [BBP] have developed stationary scattering theory for the surface Maryland model.

\section{Surface states and spectra}

The surface states of the model (2.1) are the wave packets which remain localized near the boundary $\partial \mathbb{Z}_{+}^{d+1}$ for all time. This heuristic description can be made mathematically rigorous as follows.

We say that a vector $\psi$ is a surface state of the operator $H$ if

$$
\lim _{R \rightarrow \infty} \liminf _{T \rightarrow \infty} \frac{1}{2 T} \int_{-T}^{T}\left\|1_{R} \mathrm{e}^{-\mathrm{i} t H} \psi\right\|^{2} \mathrm{~d} t=\|\psi\|^{2} .
$$

We denote the set of all surface states by $\mathcal{H}_{\mathrm{s}}(H) . \quad 1_{\Theta}(H)$ denotes the spectral projection of $H$ onto the Borel set $\Theta$.

Theorem 6.1 [JL3]

(i) $\mathcal{H}_{\mathrm{s}}(H)$ is a closed subspace of $\mathcal{H}$ invariant under $H$.

(ii) $\operatorname{Ran} 1_{\mathbb{R} \backslash \sigma\left(H_{0}\right)}(H) \subset \mathcal{H}_{\mathrm{s}}(H)$.

Part (i) of this theorem allows to define the surface spectrum of the operator $H$, $\sigma_{\mathrm{s}}(H)$, by

$$
\sigma_{\mathrm{s}}(H)=\sigma\left(H \uparrow \mathcal{H}_{\mathrm{s}}(H)\right) .
$$

Part (ii) yields that $\sigma(H) \backslash \sigma\left(H_{0}\right) \subset \sigma_{\mathrm{s}}(H)$.

An important question is whether $H$ has some surface spectra on $\sigma\left(H_{0}\right)$. The only known examples where this happens are periodic potentials [DS] and some special surface potentials for which $H$ has embedded eigenvalues in $\sigma\left(H_{0}\right)$ [MV1].

Theorem 6.2 [JL3] Assume that the wave operators $\Omega^{ \pm}$are complete on a Borel set $\Theta$. Then $\sigma_{\mathrm{s}}(H) \cap \Theta=\emptyset$. In particular, $\sigma_{\mathrm{s}}(H) \subset \mathfrak{R}(H)$.

We remark that Theorems 6.1 and 6.2 hold for an arbitrary surface potential $V$.

Surface states in $\mathcal{H}_{\mathrm{ac}}(H)$ are called surface waves. Surface waves are localized near the boundary $\partial \mathbb{Z}_{+}^{d+1}$ and propagate along this boundary. For symmetry reasons surface waves exist if the surface potential $V$ is periodic. An explicit example is a 
constant surface potential $V=a$, with $|a|>1$. In this case the spectrum of $H$ is purely absolutely continuous and $\sigma_{\mathrm{s}}(H)=[-2 d, 2 d]+a+a^{-1}$. An important question is whether surface waves exist for quasi-periodic and random surface potentials.

In what follows we consider random surface potentials. For simplicity, we assume that the random variables $\{V(n)\}$ are i.i.d. with distribution $\mathrm{d} \mu=p(x) \mathrm{d} x$. As we have described in Section 4, in many situations the spectrum of $H$ outside $\sigma\left(H_{0}\right)$ is $P$-a.s. pure point and hence in these regimes there are no surface waves with energies outside $\sigma\left(H_{0}\right)$. The question whether in some situations there is some absolutely continuous spectrum outside $\sigma\left(H_{0}\right)$ is the problem of Anderson delocalization (see [JM3] for a discussion). This fundamental problem is very little understood. On the other hand, by Theorem 4.1, a surface state with energy in $\sigma\left(H_{0}\right)$ is automatically a surface wave, and it is natural question whether for random potentials there are any surface spectra on $\sigma\left(H_{0}\right)$.

The only existing result in this direction is negative. We state the result of [JL3] under a simple assumption on the density $p$, see [JL3] for details and additional information. We use the symbol $\mathbb{E}(f)$ for the expectation of a random variable $f$.

Theorem 6.3 [JL3] Assime that $d+1=2$ and let $H=H_{0}+\lambda V, V \in \Omega$, where $\lambda$ is a real constant. Assume that $p(x)=O\left(\langle x\rangle^{-\alpha}\right)$ for some $\alpha>5 / 3$. Then there are constants $0<a<b$ such that for $|\lambda| \notin[a, b], R \geq 0$ and $\psi \in \mathcal{H}$,

$$
\lim _{T \rightarrow \infty} \frac{1}{2 T} \int_{-T}^{T} \mathbb{E}\left(1_{R} \mathrm{e}^{-\mathrm{i} t H} 1_{\sigma\left(H_{0}\right)}(H) \psi \|^{2}\right) \mathrm{d} t=0 .
$$

In particular, for $|\lambda| \notin[a, b], \sigma_{\mathrm{s}}(H) \cap \sigma\left(H_{0}\right)=\emptyset$.

The proof of this theorem combines Aizenman-Molchanov theory [A, AM] with techniques of scattering theory. More precisely, we use Aizenman-Molchanov theory to prove a "localization" estimate for matrix elements of the resolvent $(H-z)^{-1}$ along the boundary $\partial \mathbb{Z}_{+}^{d+1}$. Such an estimate implies that propagation of wave packets along the boundary is supressed. We then combine this estimate with techniques of scattering theory to show that wave packets with energies in $\sigma\left(H_{0}\right)$ must dissolve in the bulk and that the estimate (6.1) holds. A perhaps surprising aspect of this argument is that Aizenman-Molchanov theory is used to establish a result which is in spirit opposite to "localization" of wave packets.

The reason we need $d+1=2$ is related to use of Aizenman-Molchanov theory and is discussed in detail in [JL3]. We do not know whether this restriction is technical or new physical phenomena emerge in dimensions $d+1>2$.

\section{References}

[A] Aizenman M.: Localization at Weak Disorder: Some Elementary Bounds, Rev. Math. Phys., 6, 1163 (1994).

[AM] Aizenman M., Molchanov S.: Localization at Large Disorder and at Extreme Energies: An Elementary Derivation, Commun. Math. Phys. 157, 245 (1993). 
[BBP] Bentosela F., Briet Ph., Pastur L.: In preparation.

[CS] Chahrour A., Sahbani J.: On the Spectral and Scattering Theory of the Schrödinger Operator with Surface Potential, Rev. Math. Phys. 12, 561 (2000).

[CFKS] Cycon H., Froese R., Kirsch W., Simon B.: Schrödinger Operators. Springer-Verlag, Berlin-Heidelberg 1987.

[G] Grinshpun V.: Localization for Random Potentials Supported on a Subspace, Lett. Math. Phys. 34, 103 (1995).

[GJMS] Gordon Y., Jakšić V., Molchanov S., Simon B.: Spectral Properties of Random Schrödinger Operators with Unbounded Potentials, Commun. Math. Phys. 157, 23 (1993).

[DJ] Derezinski J., Jakšić V.: Spectral Theory of Pauli-Fierz Hamiltonians, J. Funct. Anal. 180, 243 (2001).

[DS] Davies E.B., Simon B.: Scattering Theory for Systems with Different Spatial Asymptotics on the Left and Right, Commun. Math. Phys. 63, 277 (1978).

[FP] Figotin A., Pastur L.: An Exactly Solvable Model of Multidimensional Incommensurate Structure, Commun. Math. Phys. 95, 410 (1984).

[JL1] Jakšić V., Last Y.: Corrugated Surfaces and A.C. Spectrum, Rev. Math. Phys. 12, 1465 (2000).

[JL2] Jakšić V., Last Y.: Spectral Structure of Anderson Type Hamiltonians, Invent. Math. 141, 561 (2000).

[JL3] Jakšić V., Last Y.: Surface States and Spectra, Commun. Math. Phys. 218, 459 (2001).

[JM1] Jakšić V., Molchanov S.: On the Spectrum of the Surface Maryland Model, Lett. Math. Phys. 45, 185 (1998).

[JM2] Jakšić V., Molchanov S.: On the Surface Spectrum in Dimension Two, Helv. Phys. Acta 71, 629 (1999).

[JM3] Jakšić V., Molchanov S.: Localization of Surface Spectra, Commun. Math. Phys. 208, 153 (1999).

[JM4] Jakšić V., Molchanov S.: Wave Operators for the Surface Maryland Model, J. Math. Phys. 41, 4452 (2000).

[JMP] Jakšić V., Molchanov S., Pastur L.: On the Propagation Properties of Surface Waves, Wave Propagation in Complex Media, IMA Vol. Math. Appl. 96, 143 (1998). 
[KMP] Kirch W., Molchanov S., Pastur L.: One-dimensional Schrödinger Operator with Unbounded Potential, Func. Anal. Prilozhen. 24, 14 (1990).

[KP] Khoruzenko B.A., Pastur L.: The Localization of Surface States: An Exactly Solvable Model, Physics Reports 288, 109-126 (1997).

[M1] Molchanov S.: Lectures given at Caltech, Spring 1990.

[M2] Molchanov S.: Lectures on Random Media. In Lectures on Probability, ed. P. Bernard, Lecture Notes in Mathematics, 1581, Springer-Verlag, Heidelberg 1994.

[MV1] Molchanov S., Vainberg B.: unpublished.

[MV2] Molchanov S., Vainberg B.: private communication.

[SW] Simon B., Wolff T.: Singular Continuous Spectrum Under Rank One Perturbations and Localization for Random Hamiltonians, Commun. Pure Appl. Math. 39, 75 (1986).

Department of Mathematics and Statistics

MCGILL UNIVERSITY

805 Sherbrooke Street West

Montreal, QC, H3A 2K6, Canada

jaksic@math.mcgill.ca 